\title{
Effect of machine hammer peening on the surface integrity of a ZnAl-based corrosion protective coating
}

\author{
Alina Timmermann ${ }^{1, *}$, Mohamed Abdulgader ${ }^{2}$, Leif Hagen $^{2}$, Alexander $\mathrm{Koch}^{3}$, Philipp \\ Wittke $^{3}$, Dirk Biermann ${ }^{1}$, Wolfgang Tillmann ${ }^{2}$, and Frank Walther ${ }^{3}$ \\ ${ }^{1}$ Institute of Machining Technology (ISF), TU Dortmund University, Baroper Str. 303, 44227 \\ Dortmund, Germany \\ ${ }^{2}$ Institute of Materials Engineering (LWT), TU Dortmund University, Leonhard-Euler-Str.2, 44227 \\ Dortmund, Germany \\ ${ }^{3}$ Department of Materials Test Engineering (WPT), TU Dortmund University, Baroper Str. 303, \\ 44227 Dortmund, Germany
}

\begin{abstract}
Thermally sprayed protective coatings are applied onto many mechanically stressed components such as support structures, shafts, turbine blades or heat exchangers. In addition to the static or cyclic load, a superimposition with corrosion processes occurs in many cases. Thermal sprayed $\mathrm{ZnAl}$ coatings are known for their performant corrosion protection properties. Within this context, the potential of $\mathrm{ZnAl}$-based layer systems was analyzed regarding corrosion fatigue behavior. Therefore, a time- and cost-efficient testing strategy based on a corrosion-superimposed load increase procedure was used to estimate the effects of a corrosive attack during cyclic loading. The investigated coating systems were thermally sprayed and partially post-processed with a Machine Hammer Peening (MHP) operation. This treatment was identified as an appropriate technique for compressing and smoothing coated surfaces. The inter-relationships between the parametrization of the MHP process, the resulting surface integrity, and the estimated corrosion fatigue properties were analyzed. The investigations indicate a positive effect of MHP post-processing operations on the surface properties of the ZnAl-based coating system.
\end{abstract}

\section{Introduction}

Offshore structures such as offshore wind plants are exposed to strong corrosive influences (see Fig. 1) with difficult conditions for repair and maintenance. In addition to cathodic corrosion protection (protective current), the application of corrosion protection coatings is an option [1-2]. For the design of optimized protective coatings in offshore applications, the consideration of mechanical resistance is necessary due to the influence of the foundation's weight, the water and wind force [3]. Therefore, appropriate measurement techniques are required to enhance the mechanical properties of the corrosion protected structures.

\footnotetext{
* Corresponding author: alina.timmermann@tu-dortmund.de
} 


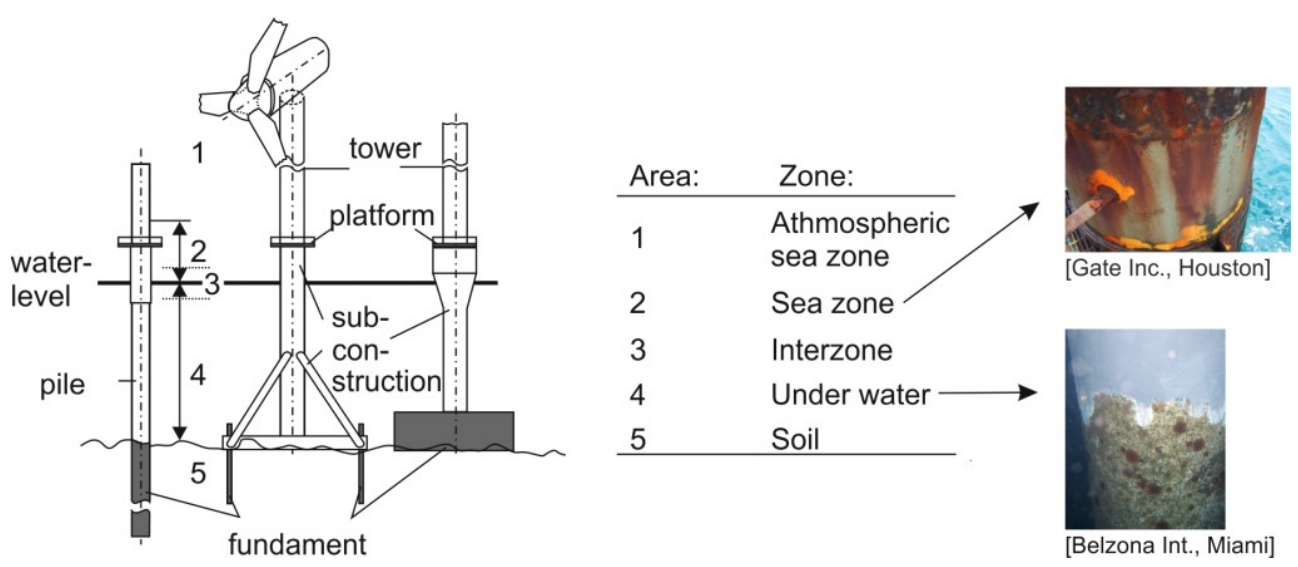

Fig. 1 Corrosion zones of an offshore wind energy plant [1]

ZnAl-based coatings have become an established option to protect steel structures, buildings and offshore wind turbines against corrosion. The superior corrosion protection properties of $\mathrm{ZnAl}$ based coatings are mainly assigned to the formation of hydrozincite or simonkolleite, as well as alumina as a resulting corrosion product [1]. ZnAl-based coatings are commonly deposited using the twin wire arc spraying (TWAS) process [4-5]. , TWASsprayed coatings are characterized by a heterogeneous lamellar microstructure with an increased amount of pores and rough coating surface. In the as-sprayed state, the obtained coatings also exhibit a high surface roughness, which reduces its resistance against corrosion under cycling loading.

An improvement of the corrosion resistance requires the use of appropriate posttreatments, such as Machine Hammer Peening (MHP). This procedure has demonstrated the potential for densifying the microstructure and smoothening surface asperities of thermally sprayed coatings [6]. In the field of surface engineering using thermal spray technology, MHP presents a novel technological approach for modifying the surface integrity of the coating.

Nevertheless, MHP became an established post-processing method for increasing the mechanical resistance of thermally sprayed wear protection coatings [6-7]. MHP involves hammering against the surface of e.g. a metallic work piece with an impact tool in defined path distances. The hammering induces kinetic energy and thus generates densification and smoothening effects of the coated surface and compressive residual stresses as well as strain hardening effects due to plastic deformation [7-8].

In order to estimate the corrosion fatigue behavior in a time and cost-efficient way, corrosion superimposed load increase tests were used, see [9]. Therefore, different measurement techniques were applied in order to measure the material response on the stepwise increased load and the superimposed corrosion. The material response can give additional information about the fatigue progress, so that a detailed estimation of the fatigue properties, especially the fatigue strength as well as the whole fatigue progress gets possible [10-11].

In this research project, a novel, interdisciplinary approach is investigated to post-process arc-sprayed $\mathrm{ZnAl}$ corrosion protection coatings by using MHP. Surface quality and corrosive-mechanical resistance of the generated hybrid material are the main objects of study. 


\section{Material and experimental procedure}

The process chain, which is applied for the experiments (Fig. 2), included the application of the corrosion protection layer, the MHP post-treatment as well as corrosion fatigue tests for the evaluation of surface quality and corrosive-mechanical resistance. Flat specimens were processed for evaluating the surface integrity, while round specimens were used for the fatigue tests.

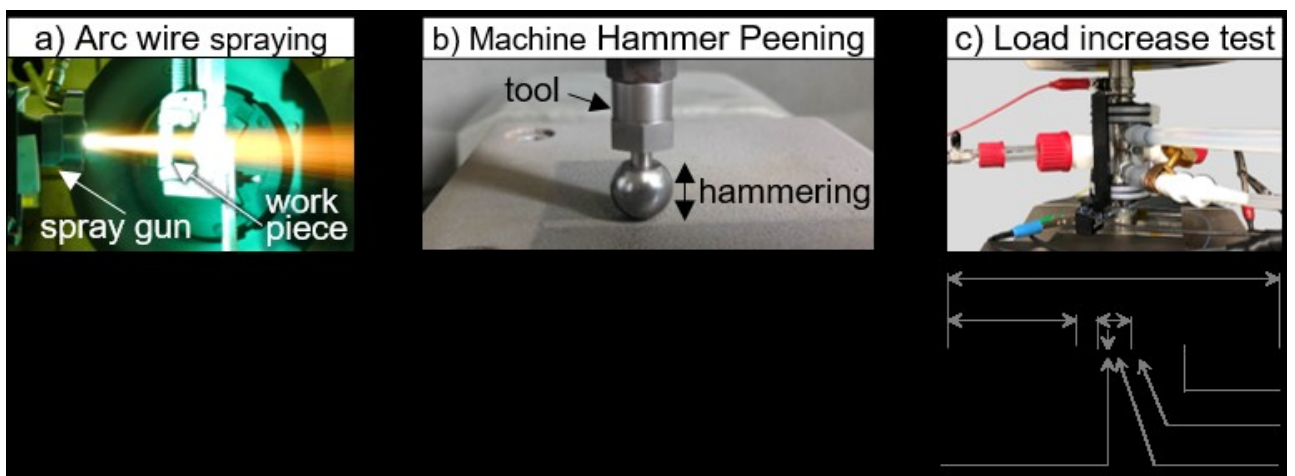

Fig. 2 Experimental set-up: Arc wire spraying (a), Machine Hammer Peening post treatment (b) and load increase test under corrosive conditions with specimen's geometry (c)

\subsection{Layer application and post-treatment}

The sandblasting pre-treatment of planar C45 (AISI 1045) steel was carried out using alumina oxide powder with EKF 24, a blasting pressure of 4 bar, $100 \mathrm{~mm}$ stand-off distance, and a blasting angle of $45^{\circ}$. The TWAS-coating was done using Durum Durspray 450 by Durum GmbH, Zn5Al solid wire as feedstock, and an industrial robot system type IRB 4600 (ABB Ltd) which was used to allow the processing of steady coating thickness.

The pneumatically operated FORGEFix Air tool (3S-engineering $\mathrm{GmbH}$ ) was applied for the MHP process. The MHP tool with a ball diameter of $d_{\mathrm{b}}=10 \mathrm{~mm}$ consisting of cemented carbide, was integrated into the Deckel Maho DMU 50 eVolution CNC machine tool. The process parameters tool feed velocity $v_{\mathrm{f}}$ and the applied tool working pressure $p$ were each varied in two stages.

\subsection{Surface measurement and cyclic testing}

Shape and roughness of the ZnAl-coated, MHP post-treated surface characteristics were measured using the 3D profilometer InfiniteFocus (Alicona). Cross-sections of the specimens were examined including metallographic shape, porosity and microhardness properties. For this purpose, a confocal white light microscope $\mu$ surf (NanoFocus) and the Nanoindenter G200 (Agilent) were applied.

For the investigation of the fatigue behavior under consideration of the corrosive influence, instrumented load increase tests were carried out using a Schenck PSB100 with Instron 8800 controller servohydraulic testing system equipped with a load cell with a load capacity of $100 \mathrm{kN}$ under corrosive conditions in a $3.5 \% \mathrm{NaCl}$ solution as well as air for comparison reasons. The specimens were tested using a stress ratio of $R=0.1$ and a test frequency of $f=10 \mathrm{~Hz}$ with a triangular load-time function at room temperature. Additional measurement techniques such as an extensometer, a thermocouple and a three-electrode arrangement were used to follow the materials responses. 


\section{Results and Discussion}

\subsection{Treatment of the $\mathrm{ZnAl}$ layer with MHP}

Surface appearance as well as averaged roughness depth values Rz are shown in Figure 3 for the as-sprayed condition and the MHP-treated coating with MHP-tool feed velocities of $v_{\mathrm{f}}=250 \mathrm{~mm} \cdot \mathrm{min}^{-1}$ and $v_{\mathrm{f}}=1000 \mathrm{~mm} \cdot \mathrm{min}^{-1}$. Apparently, profile peaks, total profile height and thus the surface roughness decreased in comparison to the sprayed condition, particularly for a low feed velocity of $v_{\mathrm{f}}=250 \mathrm{~mm} \cdot \mathrm{min}^{-1}$, probably due to the extended contact time. This tendency could also be observed in the context of the MHP treatment of the wear protection layers $[6,12]$. For $v_{\mathrm{f}}=1000 \mathrm{~mm} \cdot \mathrm{min}^{-1}$, defects in form of delamination of the coating occurred. These results indicate that MHP is suitable for the treatment of the $\mathrm{ZnAl}$ coating system, if appropriate parameters are applied.

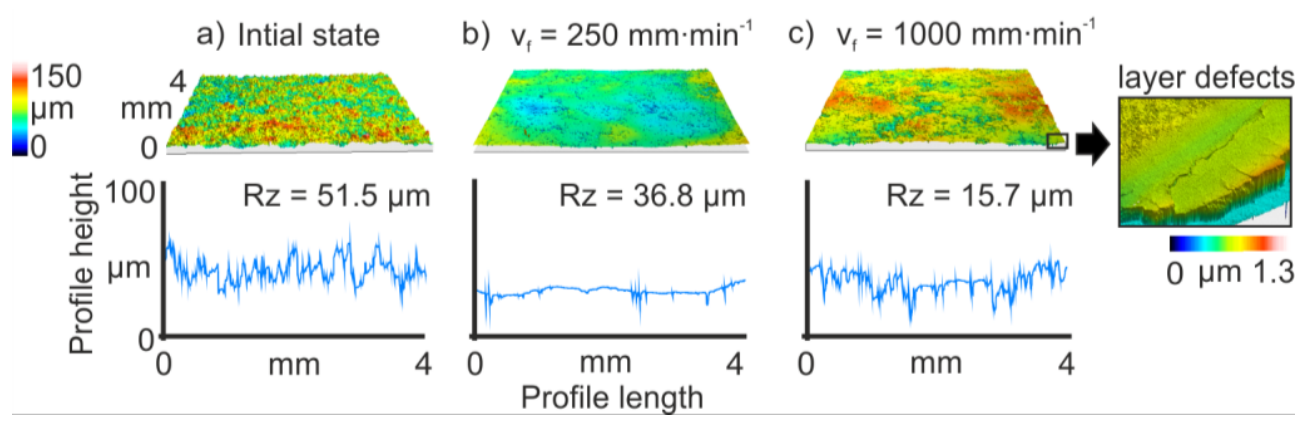

Fig. 3 Surface shape and roughness of sprayed condition (a), sprayed and MHP-treated with feed velocity of $v_{\mathrm{f}}=250 \mathrm{~mm} \cdot \mathrm{min}^{-1}(\mathrm{~b})$ and $v_{\mathrm{f}}=1000 \mathrm{~mm} \cdot \mathrm{min}^{-1}$ with a section of layer damage (c)

In Figure 4, cross-sections of the sprayed condition and the MHP-treated ZnAl layer systems under varied tool pressure of $p=4$ and 6 bar are shown. At low working pressure of $p=4$ bar, the porosity in the sub-surface was significantly reduced from about 7 to $1 \%$ and the microhardness increased from 47 to $57 \mathrm{HV} 0.01$. The observed compression of the microstructure and increase in hardness is similar to MHP post-treatment results of wear protection layers [7]. At a higher working pressure of $p=6$ bar, the effective compression depth in the coating layer increased, although the porosity and microhardness is similar.

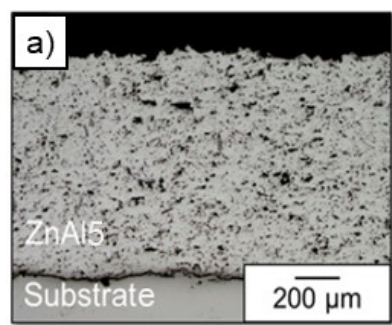

Area

Porosity (Po) in \%

Microhardness (MH) in HVO.01

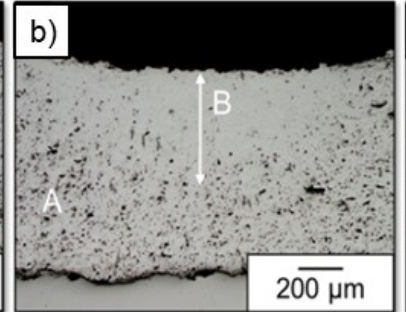

A

$7.1 \pm 0.5$ $47.6 \pm 1.4$
B

$0.7 \pm 0.1$

$56.9 \pm 4.7$

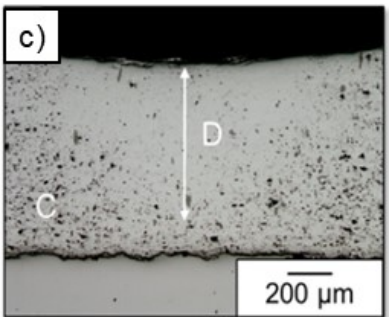

C

$6.2 \pm 0.6 \quad 0.8 \pm 0.1$

$47.6 \pm 2.5$

$56.1+2.4$

Fig. 4 Light microscopic cross-section analysis of sprayed condition (a), MHP-treated specimen with low working pressure $p=4$ bar (b) and high working pressure $p=6$ bar (c) 


\subsection{Testing of the corrosive-mechanical resistance}

Figure 5a shows the corresponding load increase test (LIT) in a $3.5 \% \mathrm{NaCl}$ solution as a function of the number of load cycles $N$ for the ZnAl-coated and MHP-treated specimen with a feed velocity of $v_{\mathrm{f}}=1000 \mathrm{~m} \cdot \mathrm{min}^{-1}$ and a working pressure of $p=6$ bar. While the plastic strain amplitude, determined from stress-strain hysteresis measurements, represents the purely mechanical part of the corrosion fatigue test, the change in the open circuit potential shows the relationship between corrosion processes and strain behavior, as it refers to the area of active iron dissolution [13].

The stress amplitude was increased in intervals of $\Delta N=10^{4}$ cycles by $\Delta \sigma_{\mathrm{a}}=10 \mathrm{MPa}$, starting from $\sigma_{\mathrm{a} \text {,start }}=100 \mathrm{MPa}$, until specimen failure. The plastic strain amplitude shows values close to zero up to about $N=28 \cdot 10^{4}$ cycles, followed by an exponential increase until specimen failure at $N_{\mathrm{f}}=38 \cdot 10^{4}$ cycles or a fracture stress amplitude of $\sigma_{\mathrm{a}, \mathrm{f}}=470 \mathrm{MPa}$. The fatigue strength is estimated based on the first changes in plastic strain amplitude (see [9]) at $\sigma_{\mathrm{a}, \mathrm{E}}=370 \mathrm{MPa}$. The increase of the open circuit potential at the beginning of the test shows that the passivation properties of the specimen's surface change. The transition to the decreasing section suggests that a critical stress or strain has been reached at which crack initiation in the corrosion protective coating occurs, so that the $\mathrm{C} 45$ substrate gets in contact with the electrolyte. The exponential increase towards the end of the test can be explained by the fact that softening mechanisms predominate and macro-crack formation is present in the base material.
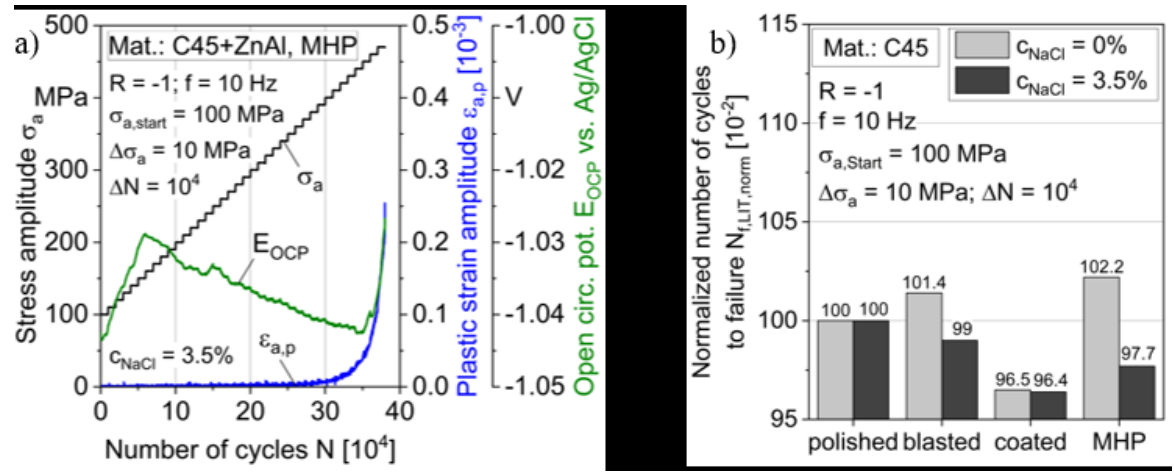

Fig. 5 Load increase test (LIT) of a ZnAl-coated MHP-treated specimen in a $3.5 \% \mathrm{NaCl}$ solution (a) and comparison of the lifetime reached in LIT for different treated specimens (b)

Figure $5 \mathrm{~b}$ compares the normalized number of cycles to failure $N_{\mathrm{f}, \mathrm{LIT} \text {, norm }}$ reached in the load increase tests under different test conditions for uncoated polished or blasted, as well as coated non-treated and MHP-treated specimens. Blasted and MHP specimens in air show slightly increased numbers of cycles to failure compared to the polished state. This can be explained by induced compressive residual stresses of the processes into the specimen, which have a positive effect on the fatigue properties [14]. Regarding tests with superimposed corrosion, all specimens achieve lower numbers of cycles to failure compared to the results in air. The electrolyte thus has an influence on the fatigue properties. The coated specimen fails first, analogous to the investigations in air. This can be explained by the roughness of the coating, which adds possible crack initiation sites to the surface. However, since both values of the coated specimens are at the same level, a protective effect against corrosive attack by the coating can be assumed. In contrast, the lifetime of the blasted and the MHPtreated specimen in the electrolyte decrease in comparison to the investigations in air. 


\section{Conclusions}

In this research, the potential of Machine Hammer Peening (MHP) for post-processing ZnAl corrosion protection coatings with the purpose of improving the corrosion fatigue behavior under mechanical loading was examined. The focus of the investigations was on the created surface quality as well as the corrosive-mechanical properties of the hybrid material evaluated by load increase tests under corrosive conditions.

The test results showed that the MHP post-treatment of the corrosion protection layer $\mathrm{ZnAl}$ is applicable without the occurrence of surface defects if appropriate parameters are applied. Reduced surface roughness, less porosity and higher hardness are potential outcomes of MHP. The load increase test for the evaluation of the mechanical behavior with superimposed corrosion showed, based on the measurement of the open circuit potential, an accumulation of microcracks in the coating surface during the test. Due to the increasing electrolyte contact with the substrate, the test specimen subsequently fails earlier. MHPtreatment of the $\mathrm{ZnAl}$ layer system increased the resistance to mechanical as well as to corrosive loads in relation to the untreated $\mathrm{ZnAl}$ layer. The ZnAl-coated specimens, MHPtreated and non-treated, demonstrated a higher resistance under mechanical, and a lower resistance under corrosive-mechanical load compared to the polished and the sandblasted reference state.

The results described were obtained within the project DFG 426365081. The authors would like to thank the German Research Foundation (DFG) for supporting the research project.

\section{References}

1. A. Momber, Materials and Corrosion, 62, p. 391-404 (2011)

2. VGB PowerTech e.V., Bundesanstalt für Wasserbau, VGB-S-021-04-2018-07-DE (2018)

3. A. Hildebrandt, A. Stahlmann, T. Schlurmann, Hansa, 2, p. 35-36 (2010)

4. F. Prenger, J. Spriestersbach, International Thermal Spray Conference and Exposition, Houston, p. 104-109 (2012)

5. D. J. Varacelle, D. P. Zeek, V. Zanchuck, E. Sampson, K. W. Couch, D. Benson, G. S. Cox, J. Therm. Spray Technol., 7(4), p. 513-520 (1998)

6. J. Wied, Ph.D. Dissertation, Technical University of Darmstadt (2011)

7. D. Biermann, P. Kersting, S. Rausch, FORM + Werkzeug 3, p. 22-23 (2015)

8. F. Lienert, J. Hoffmeister, A. Erz, V. Schulze, Proceedings of the 12th International Conference on Shot Peening (2014)

9. P. Starke, F. Walther, D. Eifler, Mater. Test., 60, 7-8, p. 669-677 (2018)

10. M. Klein, F. Walther, Materials Science Forum, 941, p. 1728-1733 (2018)

11. A. Koch, P. Wittke, F. Walther, Materials, 12(15), 2372 (2019)

12. S. Rausch, P. Wiederkehr, D. Biermann, A. Zabel, U. Selvadurai, L. Hagen, W. Tillmann, Procedia CIRP 45, p. 275-278 (2016)

13. J. Riskin, Electrocorrosion and Protection of Metals, 2, p. 213-223 (2019)

14. G. Luo, Y. Lio, Mar. Struct., 58, p. 367-381 (2018) 